\title{
Black Molecular Adsorber Coatings for Spaceflight Applications
}

\author{
Nithin S. Abraham, Mark M. Hasegawa, Sharon A. Straka \\ NASA Goddard Space Flight Center, 8800 Greenbelt Road, Greenbelt, MD 20771 USA
}

\begin{abstract}
The molecular adsorber coating is a new technology that was developed to mitigate the risk of on-orbit molecular contamination on spaceflight missions. The application of this coating would be ideal near highly sensitive, interior surfaces and instruments that are negatively impacted by outgassed molecules from materials, such as plastics, adhesives, lubricants, epoxies, and other similar compounds. This current, sprayable paint technology is comprised of inorganic white materials made from highly porous zeolite. In addition to good adhesion performance, thermal stability, and adsorptive capability, the molecular adsorber coating offers favorable thermal control characteristics. However, low reflectivity properties, which are typically offered by black thermal control coatings, are desired for some spaceflight applications. For example, black coatings are used on interior surfaces, in particular, on instrument baffles for optical stray light control. Similarly, they are also used within light paths between optical systems, such as telescopes, to absorb light.
\end{abstract}

Recent efforts have been made to transform the white molecular adsorber coating into a black coating with similar adsorptive properties. This result is achieved by optimizing the current formulation with black pigments, while still maintaining its adsorption capability for outgassing control. Different binder to pigment ratios, coating thicknesses, and spray application techniques were explored to develop a black version of the molecular adsorber coating. During the development process, coating performance and adsorption characteristics were studied. The preliminary work performed on black molecular adsorber coatings thus far is very promising. Continued development and testing is necessary for its use on future contamination sensitive spaceflight missions.

Keywords: molecular adsorber coatings, zeolite, adsorber, adsorption, stray light control, outgassing, molecular contamination, spaceflight applications

\section{INTRODUCTION}

For spaceflight applications, the phenomenon of molecular outgassing poses a significant threat to the spacecraft and the lifetime of NASA missions. This form of molecular contamination originates from materials that release molecules during orbit inside of the spacecraft. Examples of these materials include plastics, adhesives, lubricants, epoxies, tapes, potting compounds, solvents, and other similar sources. The outgassed contaminants can deposit onto hardware and instrument components, and thereby, degrade the performance of highly sensitive surfaces, such as optics, electronics, laser systems, detectors, solar arrays, and thermal control coatings. In the past, heavy honeycomb patterned ceramic pucks were used to mitigate the risk of on-orbit molecular contamination. ${ }^{1}$ However, due to the many limitations of the puck system and its mounting structures, an alternative solution for addressing the outgassing issue was necessary. ${ }^{2}$

In recent years, NASA Goddard Space Flight Center (GSFC) has developed the molecular adsorber coating (MAC) as a practical low mass and cost effective solution to address molecular contamination from outgassing. ${ }^{2}$ This sprayable, novel paint technology is comprised of processed white pigments made from highly permeable, porous zeolite minerals, and inorganic colloidal silica based binders. The combination of these components allows the coating to capture and trap outgassed materials. MAC has successfully demonstrated its technology as a coating with good adhesion performance, thermal stability, and adsorptive capability in relevant space environments. ${ }^{2}$ This innovative white coating is ready for infusion into current and future spaceflight projects and commercial markets that need to protect surfaces against the damaging effects of molecular contamination. NASA and other agencies have shown interest in this application because the technology can be directly sprayed onto most surfaces and incorporated at any phase of the mission lifetime. ${ }^{3}$ Other proposed applications include reducing pressures inside cavities with high voltage electronics, or in vacuum chambers for thermal bake-outs. 
As a white paint technology, the molecular adsorber coating offers favorable thermal control characteristics when compared to standard silicate based white thermal control coatings, such as Z-93P. However, low reflectivity properties are desired for some spaceflight applications that require specific stray light control in the near infrared range and possibly, deeper into mid to far infrared for cryogenic applications. Stray light is responsible for contrast and image quality reduction, as well as the increase of background noise for optical systems. To reduce stray light and meet specific thermal control properties, black coatings are typically applied on interior surfaces, such as instrument baffles, detectors, and high voltage electronics boxes. Black coatings are also used within light paths between sensitive optical cavities of cameras, telescopes, mirrors, and laser systems to absorb light. Examples of commonly used black polyurethane coatings include Aeroglaze ${ }^{\circledR}$ Z306 and Z307. Unfortunately, the existing molecular adsorber coating is not suitable for these spaceflight applications due to its white color. Therefore, a black version of the molecular adsorber coating was developed to provide similar adsorptive capabilities with the added benefit of reducing the effects of optical path degradation as a black coating with low reflectivity characteristics.

\section{APPROACH}

The primary objective of this study was to transform the existing white molecular adsorber coating into a black coating with similar adsorptive properties. This was accomplished with a three stage test plan, which included development, characterization, and testing. A summary of each stage is described in Table 1. The proposed test plan evaluated the overall performance of the developed black molecular adsorber coatings under ground handling environments and simulated spaceflight conditions. A comparison with the current white molecular adsorber coating was also evaluated.

Table 1. Three stage test plan for black molecular adsorber coatings

\begin{tabular}{|c|c|c|}
\hline $\begin{array}{c}\text { STAGE 1: } \\
\text { DEVELOPMENT }\end{array}$ & $\begin{array}{c}\text { STAGE 2: } \\
\text { CHARACTERIZATION }\end{array}$ & $\begin{array}{l}\text { STAGE 3: } \\
\text { TESTING }\end{array}$ \\
\hline $\begin{array}{l}\text { - Modify the current formula by } \\
\text { incorporating black pigments } \\
\text { Evaluate the changes in } \\
\text { adhesion performance of the } \\
\text { new coating formulas }\end{array}$ & $\begin{array}{l}\text { Characterize the thermal and } \\
\text { optical properties of the new } \\
\text { coatings } \\
\text { - Characterize the surface } \\
\text { morphology with microscopic } \\
\text { imaging techniques }\end{array}$ & $\begin{array}{l}\text { Evaluate the thermal } \\
\text { survivability and vacuum } \\
\text { stability of the new coatings } \\
\text { - } \\
\begin{array}{l}\text { Determine the molecular } \\
\text { capacitance of the new coatings }\end{array}\end{array}$ \\
\hline
\end{tabular}

\section{DEVELOPMENT}

\subsection{Black pigments}

In the development stage, the existing molecular adsorber coating was modified to incorporate black pigments into the formula. Several parameters, such as the type of pigment, the amount of pigment added, the binder to pigment ratios, and the changes to the method of processing or treating the pigment for compatibility were explored. As shown in Table 2, a total of 24 variations of black molecular adsorber coating formulas were developed and evaluated. For each formula, three 2" by 2" aluminum substrates were spray coated at thicknesses between 2.0 and 13.0 mils, resulting in a total of 72 samples.

Five different types of black pigments, listed in Table 2, were investigated in this study. These pigments consisted of commercially available inorganic black powders, such as specialty grade carbon black, bone charcoal, or carbon infused silica type materials. The use of polymeric based black pigments was avoided due to the possibility of it outgassing or blocking the available adsorption sites on the zeolite treated pigment. The amount, or percentage, of black pigment incorporated into the coating formula was altered between 40 and 80 percent to achieve the desired color and thermal properties that are appropriate for stray light control on optical surfaces. The binder to pigment ratios for the pigments and coatings were varied between 0.6 and 4.8 to achieve coating adhesion and proper coating viscosity for spray application. Similar processing and treatment techniques for the pigments from past studies were also executed to optimize the adhesion performance and compatibility of the coating materials. ${ }^{2}$ 
Table 2. Summary matrix of pigments, formulas, and substrates for black molecular adsorber coatings

\begin{tabular}{|ccc|}
\hline $\begin{array}{c}\text { PIGMENT } \\
\text { IDENTIFICATION }\end{array}$ & $\begin{array}{c}\text { FORMULA } \\
\text { VARIATIONS }\end{array}$ & $\begin{array}{c}\text { SAMPLE } \\
\text { SUBSTRATES }\end{array}$ \\
\hline 1-MH & 3 & 36 \\
2-ML & 12 & 18 \\
$3-\mathrm{CV}$ & & 9 \\
$4-\mathrm{CB}$ & 6 & 9 \\
5-CR & 3 & $\mathbf{7 2}$ \\
\hline Totals & $\mathbf{2 4}$ & 9 \\
\hline
\end{tabular}

\subsection{Adhesion performance}

The adhesion performance of black molecular adsorber coatings was measured in accordance with Method A of the ASTM D3359-02 standard test method. This adhesion tape test evaluated signs of delamination or separation of the coating after spray application. Some factors that may affect coating adhesion are its formulation, thickness, and spray application method. Analysis of the tape specimens showed that many of the black pigment infused formulas had failures on the top layer of the coating, rather than at the substrate interface. Failures occurring at the top layer may suggest that the cohesive bond between the top and underlying layer of the coating are slightly weak. Consequently, it was difficult to appropriately determine the adhesive bonding strength at the coating to substrate interface.

The most promising results of the 24 coating variations derive from the formulas made with the 2-ML pigment. Although not all of the formulas using this specific pigment performed well, those that did were further investigated to select a potential candidate for characterization and testing purposes. Based on additional adhesion evaluation, the formula identified as BLACK MAC 2-ML-H75 was selected as the preliminary black coating to continue with further analysis. Although results demonstrated good adhesion, additional optimization of this coating, such as varying the amount of binder and pigments or processing techniques, may be required in the future to advance its adhesion performance.

\section{CHARACTERIZATION}

\subsection{Thermal and optical property}

In the characterization stage, thermal and optical properties were measured on samples that were spray coated with the selected BLACK MAC 2-ML-H75 formula at various coating thicknesses. The measurements included solar absorptance, normal emittance, and reflectance. Solar absorptance was calculated using the AZ Technology LPSR300 spectral reflectometer. This instrument measured the reflectance for a spectral range of 0.25 to 2.8 microns at a 15 degree angle of incidence using the ASTM E903-82 standard test method. Normal emittance was also calculated using the Gier-Dünkle DB-100 infrared reflectometer. This instrument measured the normal emittance from 5 to 40 microns at room temperature using the ASTM E408-71 standard test method. Both instruments have a measurement accuracy of \pm 0.02 for solar absorptance and normal emittance values.

Table 3. Summary of thermal and optical properties for molecular adsorber coatings and common thermal control coatings ${ }^{4}$ for spaceflight applications

\begin{tabular}{|ccccc|}
\hline $\begin{array}{c}\text { COATING } \\
\text { TYPE }\end{array}$ & $\begin{array}{c}\text { COATING } \\
\text { DESCRIPTION }\end{array}$ & $\begin{array}{c}\text { COATING } \\
\text { THICKNESS }\end{array}$ & $\begin{array}{c}\text { SOLAR } \\
\text { ABSORPTANCE }\end{array}$ & $\begin{array}{c}\text { NORMAL } \\
\text { EMITTANCE }\end{array}$ \\
\hline White Silicate & Z-93P & $4.0-5.0$ mils & 0.16 & 0.92 \\
White MAC & B-TRL6 & $4.0-5.0$ mils & 0.30 & 0.93 \\
Black MAC & 2-ML-H75 & $2.5-8.5$ mils & 0.97 & 0.92 \\
Black Polyurethane & Aeroglaze ${ }^{\circledR}$ Z306 & $2.0-3.0$ mils & 0.96 & 0.91 \\
Black Polyurethane & Aeroglaze ${ }^{\circledR}$ Z307 & $2.0-3.0$ mils & 0.97 & 0.88 \\
\hline
\end{tabular}


As listed in Table 3, the average solar absorptance and normal emittance of black molecular adsorber coatings at thicknesses between 2.5 to 8.5 mils are 0.97 and 0.92 , respectively. Figure 1 illustrates that black MAC exhibits low reflectivity properties in the near infrared, as well as high solar absorptance within the solar spectrum. As depicted in Figure 2, both solar absorptance and normal emittance do not significantly differ with various coating thicknesses. Thermal and optical properties of common black and white spaceflight thermal control coatings, such as Z306, Z307, and Z-93P, as well as the original molecular adsorber coating formula identified as WHITE MAC B-TRL6 are listed in Table 3. This data suggests that black molecular adsorber coatings are comparable to the traditionally used black polyurethane coatings for thermal and stray light control. When compared against white MAC, the characteristics of black MAC differ only with solar absorptance, but have similar normal emittance properties.

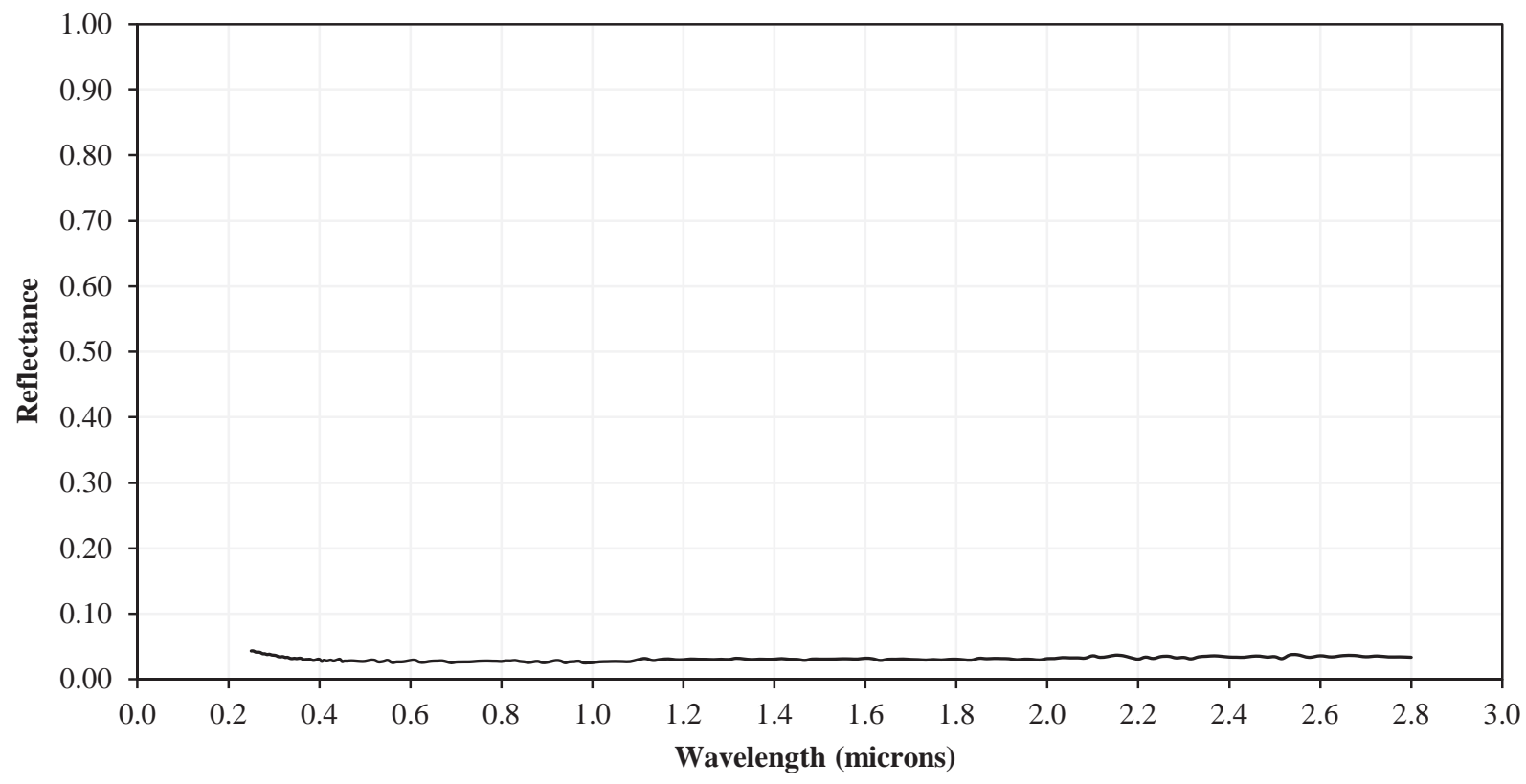

Figure 1. Reflectance curve for black molecular adsorber coatings at a thickness of 2.5 mils

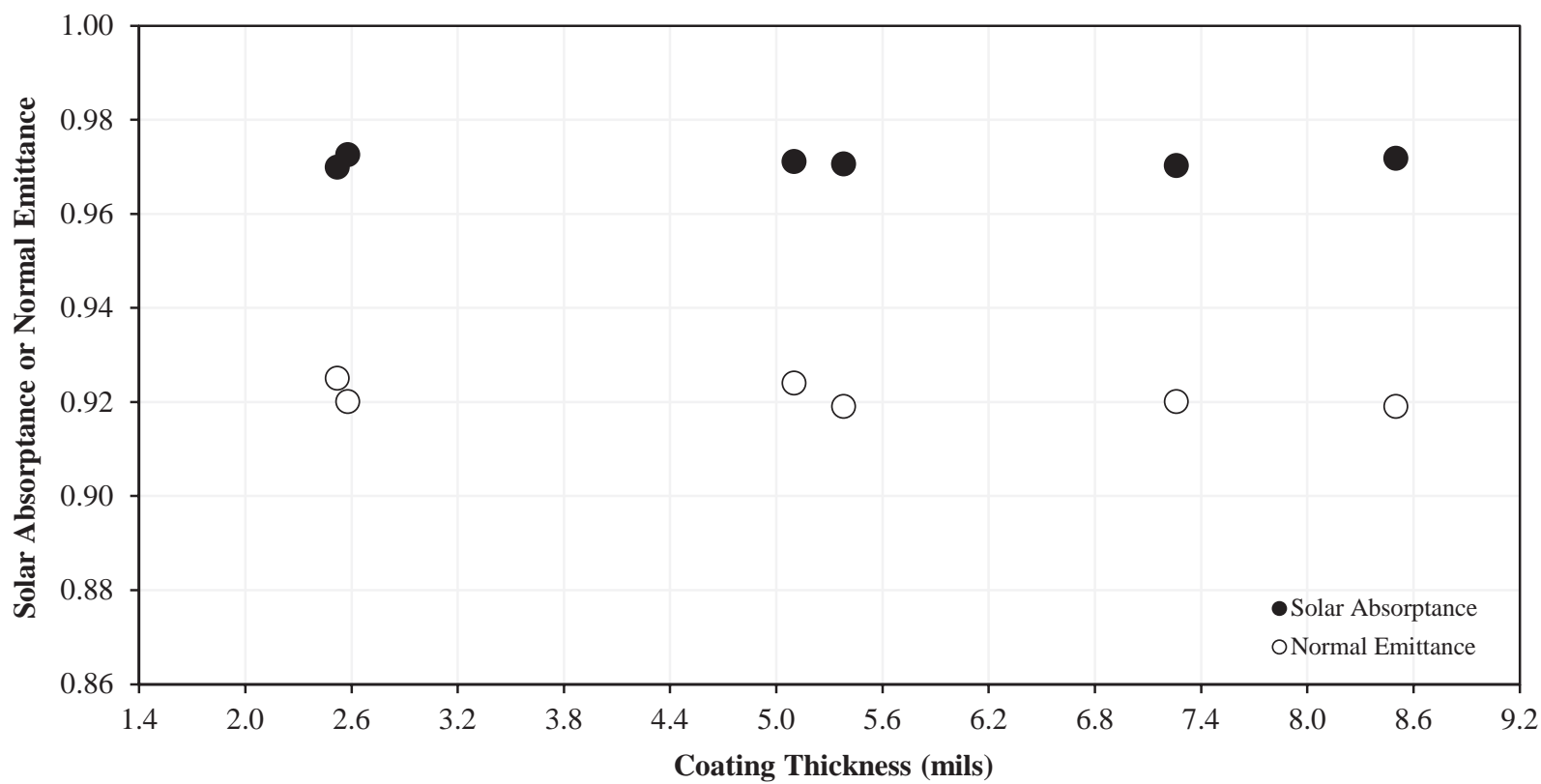

Figure 2. Effect of coating thickness on solar absorptance and normal emittance for black molecular adsorber coatings 


\subsection{Surface morphology}

Surface morphology was qualitatively evaluated on the newly developed black molecular adsorber coatings and compared against its white counterpart. This was achieved using Confocal Imaging Microscope (CIM) and Scanning Electron Microscope (SEM) analysis. For CIM analysis, an Olympus LEXT confocal laser scanning microscope was used to take three dimensional imaging of the coating surface at a 20X magnification. As depicted in Figure 3, a comparison between the two CIM images reveal that the surface morphology of black MAC appears to be flatter with less surface roughness than white MAC. This may be a result of the smaller particle size distribution of the pigments in black MAC. Further optimization of the black molecular adsorber coating formula, such as enlarging the particle size of the pigments, will help increase the surface area or texture of the coating.
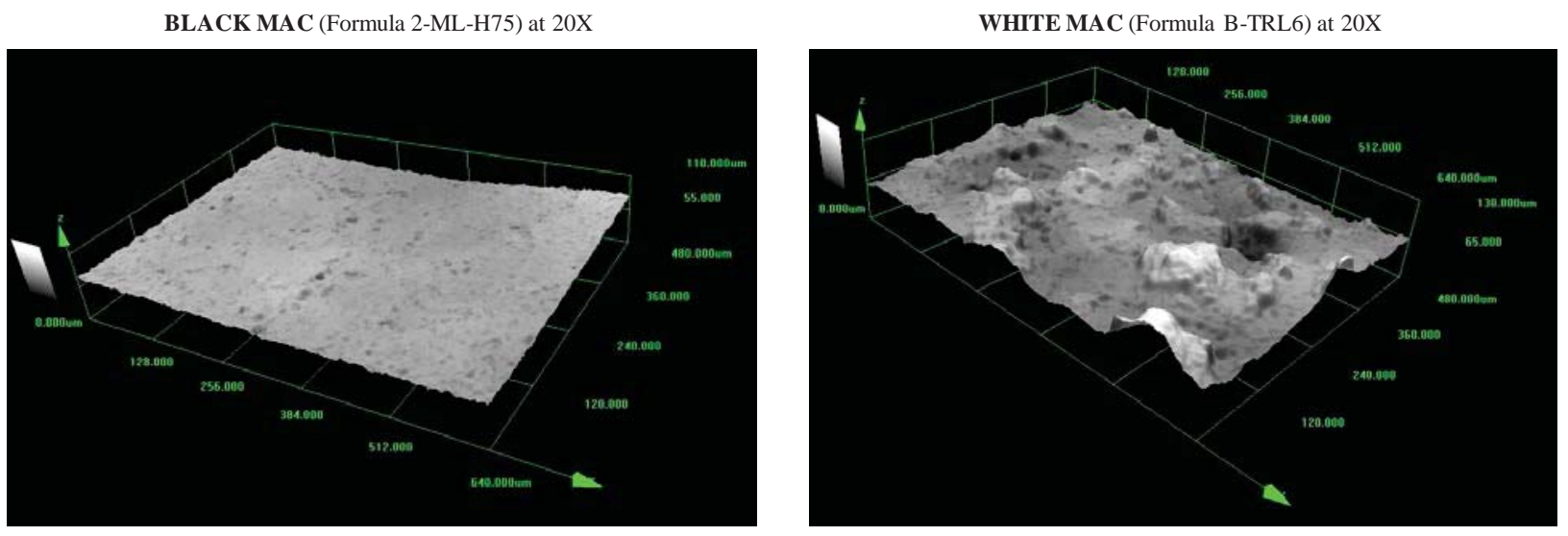

Figure 3. Comparison of CIM analysis for black and white molecular adsorber coatings

Similarly, SEM analysis was performed at a closer magnification of $456 \mathrm{X}$ at 8 kilovolts $(\mathrm{kV})$ for black MAC and 636X at 5 kilovolts for white MAC. As illustrated in Figure 4, both SEM images depict the highly porous structure of the zeolite materials in molecular adsorber coatings. The surface of black MAC shows the presence of the pores that are desired for sufficient adsorptive performance. However, these pores appear to be slighter larger, more randomly dispersed when compared to white MAC, which exhibit smaller, more uniformly dispersed pores.

BLACK MAC (Formula 2-ML-H75) at 456X at $8.00 \mathrm{kV}$ voltage

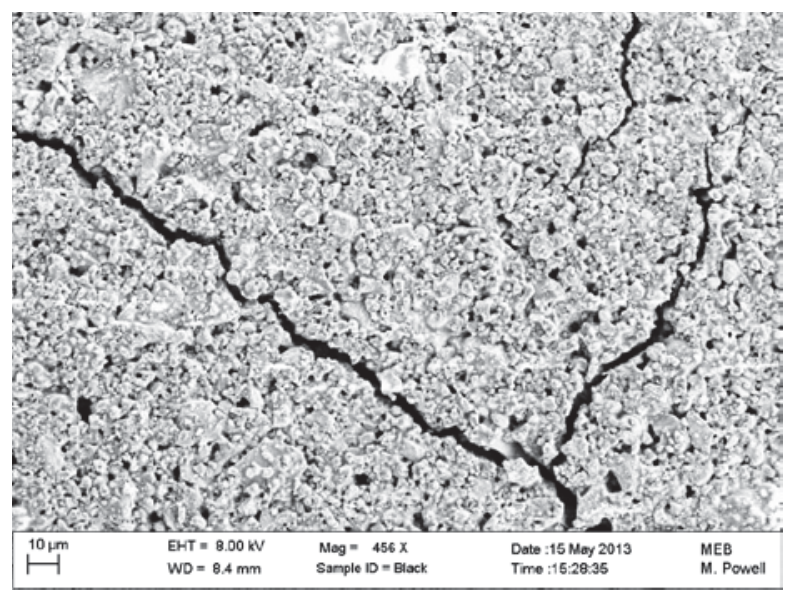

WHITE MAC (Formula B-TRL6) at $636 \mathrm{X}$ at $5.00 \mathrm{kV}$ voltage

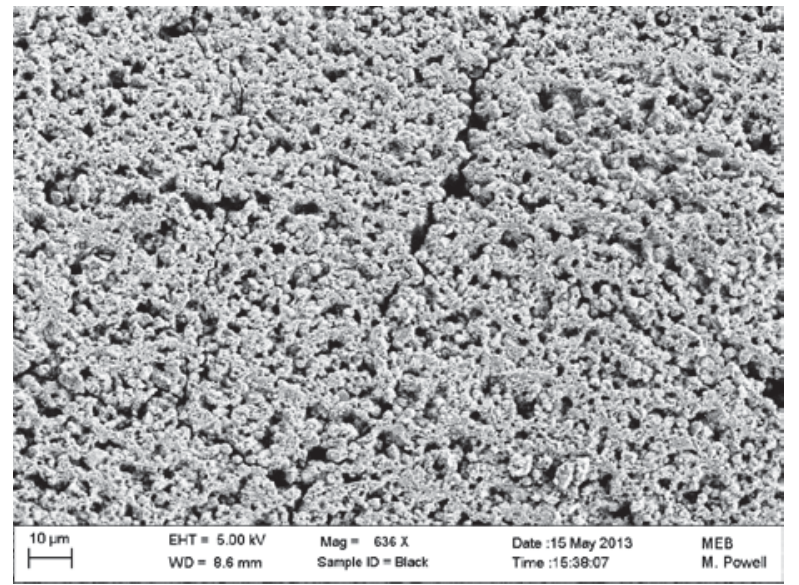

Figure 4. Comparison of SEM analysis for black and white molecular adsorber coatings 


\section{TESTING}

\subsection{Thermal survivability and vacuum stability}

Thermal cycle testing was performed to evaluate the thermal survivability and vacuum stability of black molecular adsorber coatings. The test configuration that was previously used for white MAC thermal cycle testing was replicated for the purpose of this study. ${ }^{2}$ A total of 21 samples were mounted on the thermal cycle assembly unit in a Veeco bell jar vacuum chamber. These samples consisted of the selected BLACK MAC 2-ML-H75 formula, as well as a few of the other formula variations using the 2-ML pigment. The test was operated between $-60{ }^{\circ} \mathrm{C}$ and $90{ }^{\circ} \mathrm{C}$ for approximately 100 cycles. The selected temperature parameters have a margin for both hot and cold cycles that are similar to the expected space environment. Molecular adsorber coatings are anticipated to operate at temperatures that are representative of electronics boxes and other interior surfaces, which typically reach temperatures between $-10{ }^{\circ} \mathrm{C}$ to $40^{\circ} \mathrm{C}$.

The samples were evaluated based on coating adhesion performance before and after exposure to test conditions. Results indicate that the BLACK MAC 2-ML-H75 coated samples demonstrated the most favorable results for thermal and vacuum durability. The samples had a minimal amount of coating removal after testing when compared against the other 2-ML variations. As a result, it is recommended that this coating undergo further testing under vacuum conditions for repeatability purposes, as well as after additional formula optimization.

\subsection{Molecular capacitance}

Molecular capacitance, or the coating's adsorption capability, was tested using the copper apparatus and test configuration described in a previous study. ${ }^{2}$ Multiple test runs were performed using black and white MAC samples at various coating thicknesses. A total of 18 black samples of the selected BLACK MAC 2-ML-H75 formula and 21 white samples of the original WHITE MAC B-TRL6 formula were exposed to a model contaminant source at $45^{\circ} \mathrm{C}$ for approximately 88 to 160 hours. The selected source was stearyl alcohol because it is representative of commonly outgassed hydrocarbons. Although black MAC showed less surface area based on coating morphology, preliminary molecular capacitance test data exhibit similar trends for adsorption when compared to its white coating counterpart. As shown in Figure 5, black MAC adsorbed 0.5 to $4.2 \mathrm{mg} / \mathrm{cm}^{2}$ depending on variations in thickness between 1.8 and 12.0 mils. Similarly, white MAC captured about 0.6 to $4.8 \mathrm{mg} / \mathrm{cm}^{2}$ of contaminants for thicknesses between 2.3 and 10.7 mils. Figure 5 depicts that the molecular capacitance is a function of coating thickness. For example, a white MAC sample of 6 mils is projected to have a molecular capacitance three times greater than a 3 mil sample.

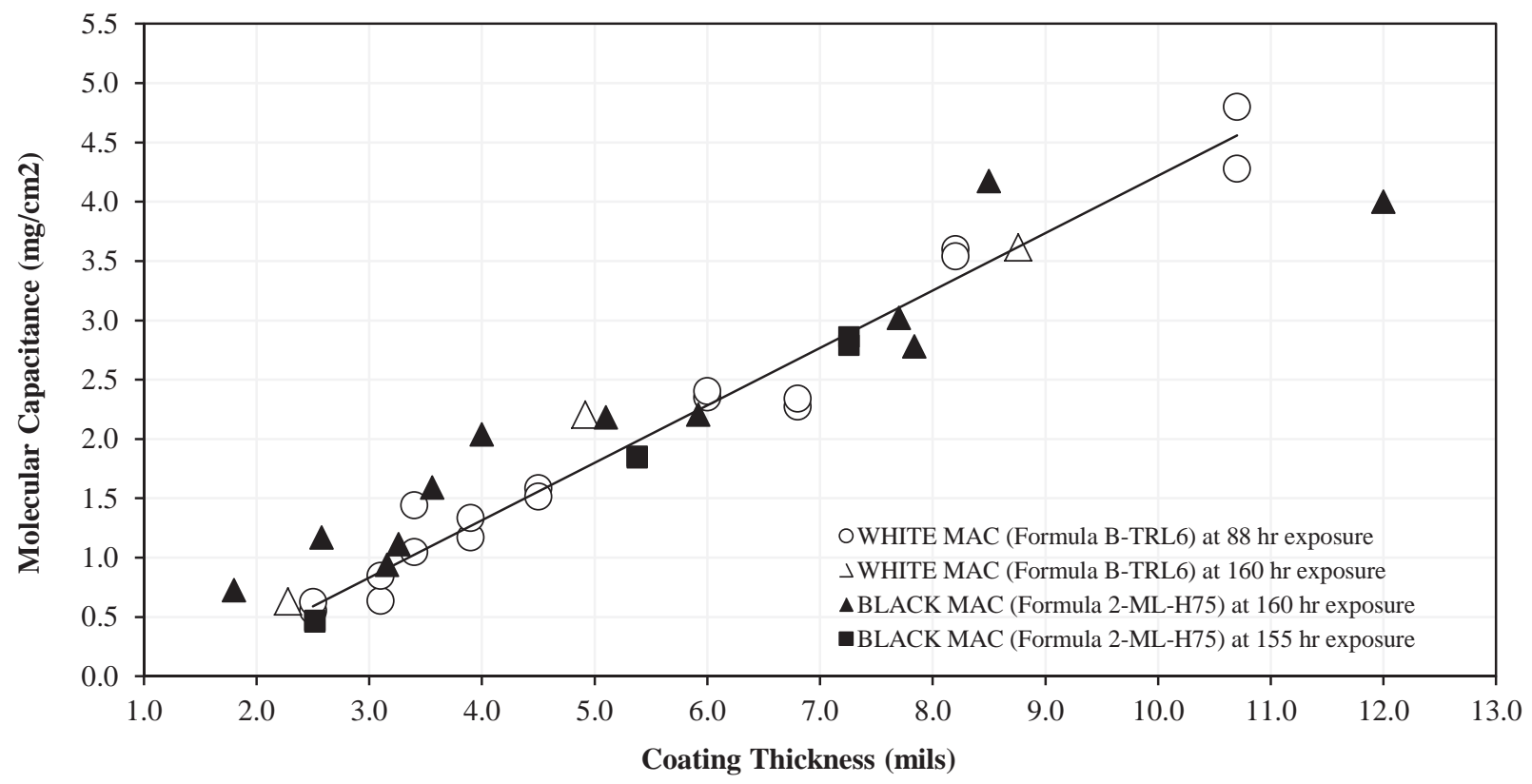

Figure 5. Effect of coating thickness on molecular capacitance for black and white molecular adsorber coatings 


\section{CONCLUSIONS}

\subsection{Summary}

In conclusion, this project has successfully demonstrated the development of black molecular adsorber coatings. Preliminary coating formulations were characterized and tested for thermal and optical properties, surface morphology, adhesion performance, thermal survivability, vacuum stability, and molecular adsorption to protect sensitive surfaces from the damaging effects of outgassed materials. These results are promising and have shown that black molecular adsorber coatings have great potential to both reduce contamination levels and address thermal and stray light control issues for spaceflight applications.

\subsection{Accomplishments}

The following is a list of accomplishments for this study:

- Modified the current white MAC formula by incorporating a variety of inorganic black powders

- Optimized the formulation process by investigating different types and quantities of black pigments, binder to pigment ratios, and methods for treating pigments

- Evaluated the adhesion performance for black MAC at ambient conditions

- Characterized the thermal and optical property measurements, such as solar absorptance and normal emittance, as a function of coating thickness

- Verified the low reflectivity characteristics of black MAC for thermal and stray light control

- Established the surface morphology with microscopic imaging techniques, such as CIM and SEM

- Investigated the adhesion performance, thermal survivability, and vacuum stability of black MAC after thermal cycling at temperatures between $-60^{\circ} \mathrm{C}$ and $90^{\circ} \mathrm{C}$

- Performed preliminary molecular capacitance testing on black MAC to establish its adsorption capability

- Compared black MAC characteristics and determined its similarities and differences with white MAC, and other commonly used black spaceflight coatings

- Selected a potential black MAC formula for further development and testing on repeatability and qualification studies

\section{FUTURE WORK}

Future work on black molecular adsorber coatings includes optimizing the formula with other inexpensive black carbon materials and evaluating the particulation shedding effects of the coating. Advancing the low reflectivity properties of the coating into the mid to far infrared wavelengths for use in cryogenic applications is also desired. Other activities include improving adhesion performance and increasing surface area for the final product. A qualification effort of the optimized product will be necessary for spaceflight and commercial use on surfaces to reduce both outgassing and optical path degradation. Several tests performed in this study will be repeated for reproducibility. Lastly, electrically dissipative and conductive versions of the coating will be developed to prevent charge build up and minimize voltage gradients on sensitive spacecraft surfaces. 


\section{ACKNOWLEDGEMENTS}

The development and testing efforts for black molecular adsorber coatings was funded through the Internal Research and Development (IRAD) program at NASA GSFC. Additional assistance for characterization and testing was provided by Code 546 Contamination and Coatings Engineering Branch and Code 541 Materials Engineering Branch personnel from NASA GSFC and Stinger Ghaffarian Technologies (SGT) Corporation. These members include John Petro (NASA GSFC, 546) for spray application, Kenneth O’Connor (SGT, 546) and Alfred Wong (SGT, 546) for vacuum thermal cycle testing, and Mollie Grossman (NASA GSFC, 541) for SEM imaging.

\section{REFERENCES}

[1] Chen, P., Thomson, S., Triolo, J., and Carosso, N., "The Use of Molecular Adsorbers for Spacecraft Contamination Control,” AIP Conference Proceedings, 361 (1) (1996)

[2] Abraham, N. S., Hasegawa, M. M., and Straka, S. A., "Development and Testing of Molecular Adsorber Coatings,” Proc. SPIE 8492, 849203 (2012).

[3] Keesey, L., "Ah, That New Car Smell: NASA Technology Protects Spacecraft from Outgassed Molecular Contaminants,” NASA, 19 November 2012. <http://www.nasa.gov/topics/technology/features/outgastech.html> (1 July 2014)

[4] Kauder, L., “Spacecraft Thermal Control Coatings References,” NASA/TP-2005-212792, December 2005 